\title{
Comparison of Dengue Infection in Human Mononuclear Leukocytes with Mosquito C6/36 and Mammalian Vero Cells Using Flow Cytometry to Detect Virus Antigen
}

\author{
Farid FO von Sydow ${ }^{++}$, Marta A Santiago*, Patricia C Neves-Souza, \\ Denise IS Cerqueira, Adriana S Gouvea, Maryrose FH Lavatori, \\ Âlvaro L Bertho* Claire F Kubelka/ ${ }^{+}$
}

Departamento de Virologia *Departamento de Protozoologia, Instituto Oswaldo Cruz, Av. Brasil 4365, 21045-900 Rio de Janeiro, RJ, Brasil

Fluorescent activated cell sorter (FACS) analysis is useful for the detection of cellular surface antigens and intracellular proteins. We used this methodology in order to detect and quantify dengue antigens in highly susceptible cells such as clone C6/36 (Aedes albopictus) and Vero cells (green monkey kidney). Additionally, we analyzed the infection in vitro of human peripheral blood mononuclear leukocytes (PBML).

FACS analysis turned out to be a reliable technique to quantify virus growth in traditional cell cultures of C6/36 as well as Vero cells. High rates of infection were achieved with a good statistical correlation between the virus amount used in infection and the percentage of dengue antigen containing cells detected in infected cultures.

We also showed that human monocytes $(C D 14+)$ are preferred target cells for in vitro dengue infection among PBML. Monocytes were much less susceptible to virus infection than cell lines but they displayed dengue antigens detected by FACS five days after infection. In contrast, lymphocytes showed no differences in their profile for dengue specific immunofluorescence.

Without an animal model to reproduce dengue disease, alternative assays have been sought to correlate viral virulence with clinical manifestations and disease severity. Study of in vitro interaction of virus and host cells may highlight this relationship.

Key words: dengue virus - C6/36 -Vero cells - mononuclear leukocytes - flow cytometry

Since the beginning of the 80 s dengue fever has disseminated throughout Brazil and became endemic. During 1998-1999 the disease, which was only threatening, become alarming with 650 thousand notified cases (Brazilian National Health Foundation 1999). The infection may turn out to be asymptomatic or a mild disease, but may also develop more severe clinical manifestations allowing its classification as dengue hemorrhagic fever or dengue shock syndrome (PAHO 1994).

The pathogenesis of dengue disease is not fully understood and is considered an immunopathologic process associated with prior immune sensitization by a heterotypic virus (Halstead 1980). Dengue infection provides lifelong homotypic im-

Financial support: Fiocruz, CNPq and Colab, Brazil ${ }^{+}$Corresponding author Fax: +55-21-598.4491. E-mail: claire@gene.dbbm.fiocruz.br

${ }^{++}$Graduated student from Instituto de Microbiologia, UFRJ.

Received 9 August 1999

Accepted 14 April 2000 munity, but only transient cross-protection against other serotypes is achieved, making sequential infection possible (Pang 1987, Kliks et al. 1989).

Dengue disease is not reproduced experimentally in any animal model other than man (Halstead 1988). Several dengue virus strains were recovered from patient lymphoid organs, being mostly of macrophage origin (Bhamarapravati \& Boonyapaknavik 1966, Boonpucknavig et al. 1979, Halstead 1988). Most work using freshly isolated cells describe in vitro dengue infection of monocytes but also of fibroblasts (Kurane et al. 1992) and endothelial cells although the latter are infected at a lower rate than monocytes (Anderson et al. 1997).

Earlier studies showed a significant variation of growth among different strains of dengue in human monocytes suggesting a relationship of virus characteristics with disease severity (Morens et al. 1991). Mononuclear phagocytes have been claimed to be a preferred target for dengue replication in vivo and, therefore, the interaction of these cells with virus is a relevant issue (Halstead 1988). However, the correlation of in vitro infection with viral virulence is not well established. 
Different cell lines have been used to propagate dengue virus in culture. Viruses replicate well in cell clone C6/36 from Aedes albopictus (Igarashi 1978) mosquito and in two other mosquito lines and they are presently used as sensitive assays for virus isolation from patients (Gubler 1998). Vero and LLC-MK2 cells, both originated from monkey kidney, are also permissive cell lines (Gubler 1998).

Several techniques have been used to quantify the growth of dengue virus. They include plaque forming units assay in Vero or LLC-MK2 cells (Russell et al. 1967) but require relatively large amounts of virus and, most importantly, a defined cytopathic effect on cells to form plaques, unlike immunofluorescence, which can detect virus antigen without cell lysis. Detection of antigenic protein by immunofluorescence can be used to quantify virus by statistical analysis of antigen in serial dilutions in microtiterplates (Reed \& Muench 1938, Shoepp \& Beaty 1984) and has been used as an alternative to plaquing assays (Miagostovich et al. 1993). Quantification of viral RNA by hybridization or quantitative RT-PCR (Vordam \& Kuno 1997, Deubel 1997, Gubler 1998) are very sensitive methodologies but still very laborious, time consuming and costly.

Alternative quantitative assays for dengue virus and/or its components are required in order to identify cells permissive to virus among peripheral blood mononuclear leukocytes (PBML) and to determine virus load in patient samples. Although molecular methods as quantitative RT/PCR have been used to identify and measure virus load, quantification of viruses by immunofluorescence methods can still provide a better approach to determine frequency and type of infected cells with a relative simplicity in performance. In this regard, fluorescent activated cell sorter (FACS), provides reliable results in detection of cellular surface antigens and intracellular proteins.

Here, we standardized a technique using FACS to quantify the rate of Dengue-1 and Dengue-2 virus infection in permissive cell cultures such as clone C6/36 and Vero cells. Starting from optimal conditions of dengue detection in these cells by FACS, we analyzed the infection in vitro of freshly isolated human PBML, considered the target cell in vivo. Cell subpopulations sorted based on their CD14 bearing characteristics were evaluated for their abilities to allow dengue virus growth.

\section{MATERIALS AND METHODS}

Cell cultures - C6/36 cell clone of A. albopictus was grown as monolayers at $28^{\circ} \mathrm{C}$ on Leibovitz medium (L-15) supplemented with $200 \mathrm{mM}$ glutamin, $1 \%$ non-essential aminoacids, $19 \%$ tryptose phosphate broth, $100 \mathrm{U} / \mathrm{ml}$ penicillin, $10 \mu \mathrm{g} / \mathrm{ml}$ streptomycin and 5\% fetal calf serum (FCS).
Vero cell monolayers were grown at $37^{\circ} \mathrm{C}$ in 199 Eagle's medium supplemented with $200 \mathrm{mM}$ glutamin, $100 \mathrm{U} / \mathrm{ml}$ penicillin, $10 \mu \mathrm{g} / \mathrm{ml}$ streptomycin and 5\% FCS.

Preparation of virus stock and virus titration Dengue-virus serotype 1, strain 16007 and serotype 2 strain 16681 were provided by Dr S Halstead (Naval Medical Research Center, USA) and serotype 2, strain New Guinea 09 by Dr R Nogueira (IOC, Fiocruz, Rio de Janeiro).

Viruses were titrated by serial dilution cultures in microtiter plates and detected by immunofluorescence as described before (Schoepp \& Beaty 1984, Miagostovich et al. 1993). Virus titers were calculated as $50 \%$ tissue culture infectious dose or $\mathrm{TCID}_{50} / \mathrm{ml}$ (Reed \& Muench 1938).

Preparation of human PBML - Mononuclear leukocytes were obtained from heparinized venous blood originated from dengue seronegative adult donors. Cells were purified through gradient density centrifugation (Histopaque 1077, Sigma, USA). Cells were suspended in RPMI 1640 supplemented with $200 \mathrm{mM}$ glutamin $100 \mathrm{U} / \mathrm{ml}$, penicillin, $10 \mu \mathrm{g} / \mathrm{ml}$ streptomycin and 20\% autologous heat inactivated plasma and incubated at $37^{\circ} \mathrm{C}$ under humid atmosphere with $5 \% \mathrm{CO}_{2}$.

Infection of cell lines - All infections were performed on 24-well plates. C6/36 and Vero cells were seeded at $2 \times 10^{5}$ cells/well in medium with $5 \%$ FCS and allowed to form a monolayer for $24 \mathrm{~h}$ at respective incubators. Cell supernatant was removed and adherent cells was washed twice with medium without FCS. Wells were filled with $0.5 \mathrm{ml}$ fresh medium containing different concentrations of Dengue-1, Dengue-2 or medium. After 90 min for adsorption, extra medium was added to achieve $1 \mathrm{ml} 2 \%$ FCS. Viruses were allowed to grow for five days in incubated cultures. Tetra- to sextuplicate wells were set for each dilution and controls.

Infection of $P B M L$ - All infections were performed on 24-well plates. PBML suspended in RPMI 1640 medium supplemented with $20 \%$ autologous inactivated serum were seeded at $2 \times 10^{6}$ cells/well. After $18 \mathrm{~h}$-incubation, two kinds of medium replacement were performed at different experiments. In some experiments, adherent cells were enriched by washing away twice unattached cells before adding virus diluted with medium in $1 \mathrm{ml}$. In other experiments, medium was aspirated very carefully not allowing suspension of cells from the well bottom and virus dilution was then added. After 90 min of incubation for adsorption extra medium were added to achieve $20 \%$ autologous serum. Virus was allowed to grow for five days. Wells were set in triplicates.

Labeling of infected cell lines and double labeling of infected PBML and flow cytometry analysis - Cells were recovered by plastic microtip 
scratching with cold medium and set at $1 \times 10^{6} \%$ microtube; they were washed once with $1 \mathrm{ml}$ Dulbecco's medium prepared without $\mathrm{Ca}^{2+}$ and $\mathrm{Mg}^{2+}$ with $2 \% \mathrm{FCS}$ and $0.01 \% \mathrm{NaN}_{3}$. Surface labeling was performed directly on viable cells but intracellular staining required a fixation with $0.5 \mathrm{ml}$ cold paraformaldehyde at 4\% in Dulbecco's medium for $10 \mathrm{~min}$ and, after centrifugation $(350 \mathrm{~g}, 5 \mathrm{~min})$, membrane permeabilization was carried out with $1 \mathrm{ml}$ $0.1 \%$ saponin Dulbecco's medium with FCS and $\mathrm{NaN}_{3}$. Primary antibody purified from ascitic fluid reacting with Dengue-1 or with Dengue-2 (1:100 dilution, ATCC, USA) was incubated in a dilution of $1: 100$ with cells for $60 \mathrm{~min}$. Cells were washed $1 \mathrm{x}$ $1 \mathrm{ml}$ medium and incubated with anti-mouse Ig labeled with FITC (1:100 dilution, Sigma, USA) or TRITC (1:60 dilution, DAKO, Denmark) for $30 \mathrm{~min}$. Intracellular staining used always saponin Dulbecco's with FCS and $\mathrm{NaN}_{3}$ as washing medium but surface staining did not require saponin and was performed always under ice bath. Cells were washed once more, ressuspended in $1 \%$ paraformaldehyde and kept at $4^{\circ} \mathrm{C}$ up to 2-3 days.

Double labeling of infected PBML was performed by incubation of recovered cells with FITClabeled antibodies to CD14 (1:20 dilution, DAKO) for $45 \mathrm{~min}$ and $1 \mathrm{x} 1 \mathrm{ml}$ Dulbecco's washing prior to fixation with $4 \%$ paraformaldehyde and $0.1 \%$ saponin permeabilization. Unspecific blocking steps in PBML labeling consisted in using 5\% FCS Dulbecco's solution during all incubation steps.

Cells were acquired (10,000 events for cells lines and 5,000 for gated CD14+PBML) and analyzed on a EPICS 751 flow cytometer (Coulter Electronics, Hialeah, FL, USA).

\section{RESULTS}

Surface and intracellular detection of dengue antigen by FACS on C6/36 cells - Dengue antigens were detected in five day-infected C6/36 cells either on surface or intracellularly by FACS (Fig. 1). Intracellular staining was slightly more sensitive in detecting virus antigen than surface staining, despite a higher background reaction and more laborious procedures. There is a proportionality in the increase of virus concentration used to infect cells expressed by $\mathrm{TCID}_{50}$, which is related to the percentage of cells with positive antigen detection. The intracellular staining was chosen in order to perform further experiments.

Frequency of virus specific labeling detected by FACS in C6/36 and Vero cells infected with increasing amounts of Dengue-1 or Dengue-2 Both Dengue-1 (Fig. 2A) and Dengue-2 (Fig. 2B) infect mosquito $\mathrm{C} 6 / 36$ and mammalian Vero cells at high and approximate rates. There is a good correlation between virus amounts (expressed in

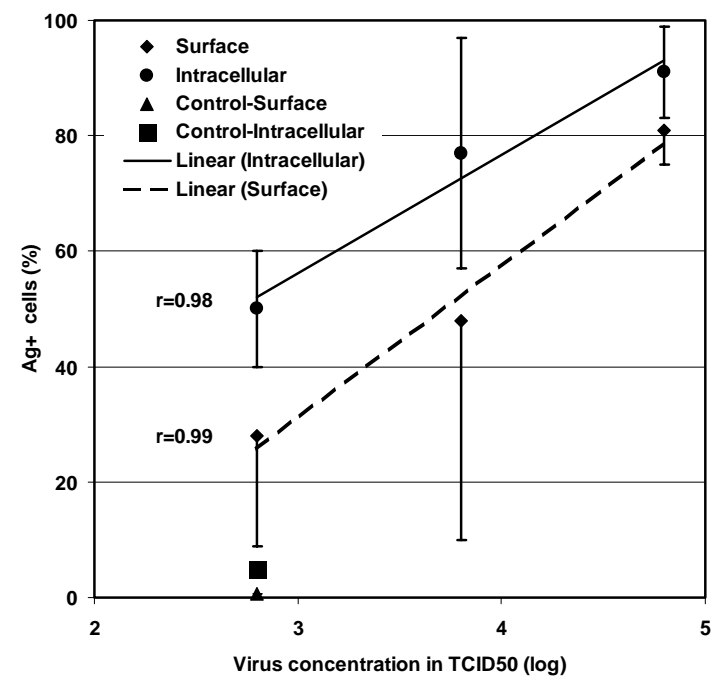

Fig. 1: Dengue-2 antigen detection by fluorescent activated cell sorter on surface or in intracellular labeled C6/36 cells. Cells were infected at different virus concentrations and after five days labeled sequentially with antibody for Dengue-2 and anti-mouse Ig-FITC. Values represent average \pm standard deviation of samples cultured in sextuplicates. The correlation coefficient $r$ was determined between virus titer in $\mathrm{TCID}_{50}$ and percentage of dengue antigen positive cells. The tendency line calculated by linear regression is represented.

$\mathrm{TCID}_{50}$ ) used to infect cells and the percentage of dengue antigen detected in infected cells after five days. Values of calculated $r$ were significant for both viruses in both cell lines.

Profiles of Dengue-1 and Dengue-2 antigens in infected PBML as determined by FACS analysis - Human PBML allow infection by dengue viruses as it is represented in Fig. 3. A well-defined Dengue-1 positive population in infected PBML is also a CD14+ population showing that monocytes are the preferred target for dengue viruses. In Fig. 4A a defined CD14+ population with large size characteristic of monocytes was gated and the histogram profile for dengue antigen confirmed the presence of a peak of cells with higher fluorescence in infected cells (Fig. 4C) than in uninfected cells (Fig. 4B). Monocyte population demonstrated homogeneity in their cells by one day culture even with treatment for intracellular labeling (data not shown). After several days in culture this population is still viable (as detected by trypan blue or propide iodine) but is extremely heterogeneous with regard to size, granularity, CD14 and dengue antigen labeling and showing always a spread pattern in plots from FACS analysis (Fig. 3A, B).

In the plot of foward scattered cells (FCS) versus side scattered cells (SSC) from Fig. 5A the popu- 
lation with small size and granularity characteristic of lymphocytes was gated. The histogram profile of gated cells showed no difference in fluorescence intensity for dengue antigen on uninfected (Fig. 5B) and infected cells (Fig. 5C), confirming the absence of infection in this cell population.

Frequencies of CD14+ and CD14- bearing for Dengue- 1 and - Dengue- 2 antigens in infected cells are represented in the Table.
Comparison of dengue infection in different cell types - The infection pattern for C6/36 is always slightly higher than Vero cells with both virus serotypes, if a tendency line is drawn by linear regression (Fig. 2).

Freshly isolated CD14+ monocytes are the target cells for dengue viruses among PBML. Nevertheless, mononuclear phagocytes (CD14+) required at least ten times higher virus concentrations than
A

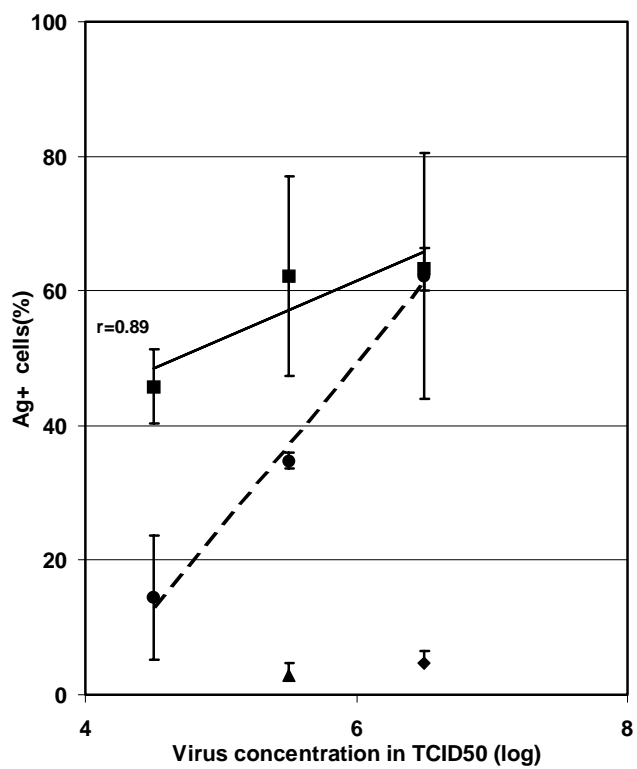

$\mathrm{C6} / 36$

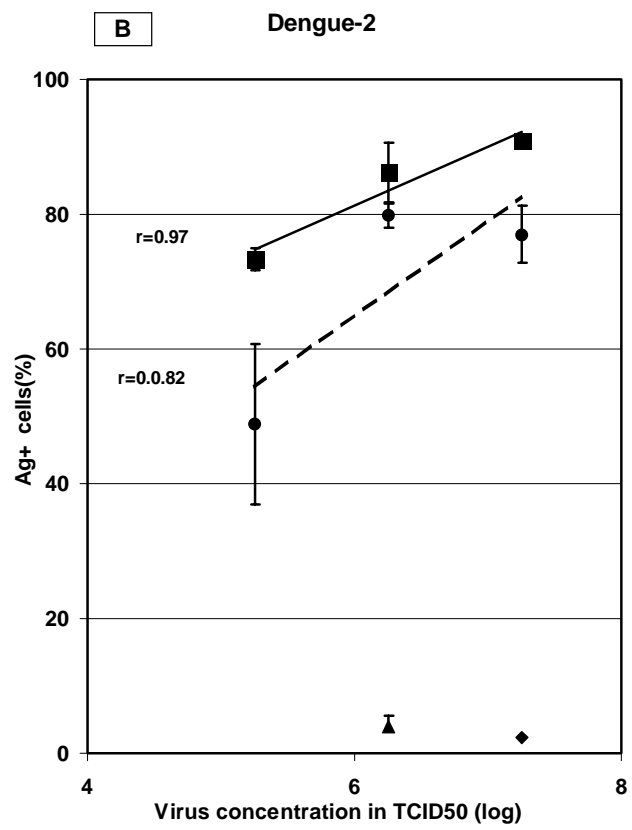

Fig. 2: dengue antigen detection by fluorescent activated cell sorter in intracellular labeled C6/36 cells or Vero cells. Cells were infected for five days using different amounts of Dengue-1 (A) or Dengue-2 (B) and labeled sequentially with antibody for Dengue-2 and anti-mouse Ig-FITC. Values represent average \pm standard deviation of samples cultured in tetraplicates. The correlation coefficient $r$ was determined between virus titer in $\mathrm{TCID}_{50}$ and percentage of dengue antigen positive cells for each virus/cell type. The tendency line calculated by linear regression is represented. Here is shown one representative experiment out of different three performed.

A

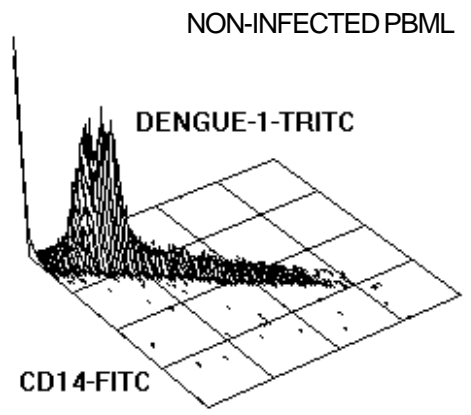

B

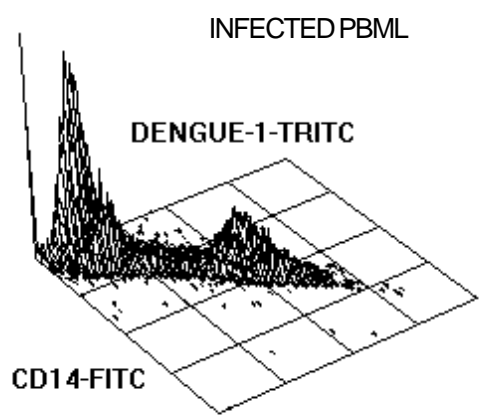

Fig. 3: 3D-contour plot from fluorescent activated cell sorter analysis of Dengue-1 infected peripheral blood mononuclear leukocytes labeled with antibodies to CD14 and Dengue-1. Total human peripheral blood mononuclear leukocytes $\left(2 \times 10^{6}\right.$ $\mathrm{ml}$ ) were set in triplicates and incubated either with medium (A) or infected for five days with $10^{6,5} \mathrm{TCID}_{50} / \mathrm{ml} \mathrm{Dengue} 1$ strain 16981 (B). Peripheral blood mononuclear leukocytes (PBML) were first labeled with anti-CD14-FITC ( $x$-axis) followed by antibody to Dengue-1 or Dengue-2 and anti-mouse Ig-TRITC ( $y$-axis). Data were collected from one experiment representative out of four using either adherent cells or total PBML cells infected with Dengue-1 or Dengue-2. 
traditional cell lines to achieve detectable antigen and much lower rates of virus labeled cells are obtained with high virus doses in inoculum. An experiment was performed inoculating cultures with less than $10^{6.5} \mathrm{TCID}_{50}$ for Dengue- 1 and less than $10^{7.25} \mathrm{TCID}_{50}$ for Dengue-2 to infect adherent

A

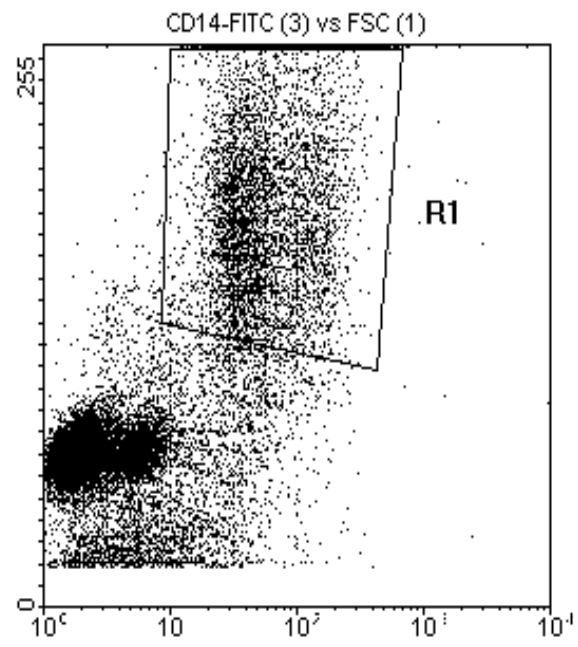

PBML and it did not produce detectable antigen by immunofluorescent methods.

\section{DISCUSSION}

Plaque forming assay is still widely used to quantify virus grown and/or infectivity (Russell et

B

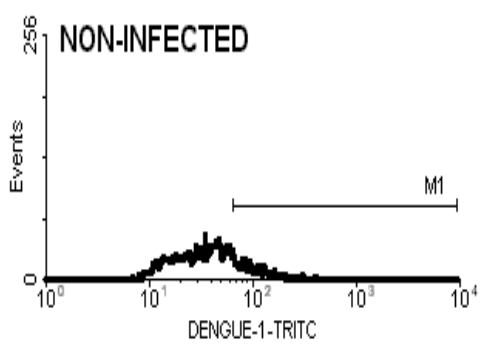

C

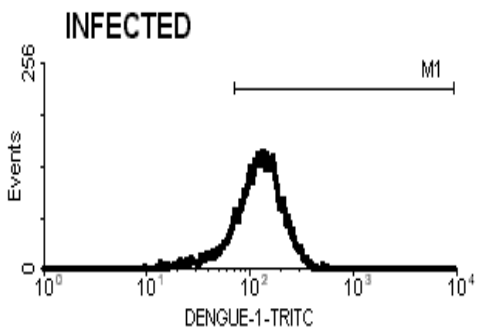

Fig. 4: histograms from gated CD14+monocytes obtained from fluorescent activated cell sorter analysis of Dengue-1 infected peripheral blood mononuclear leukocytes labeled with antibodies to CD14 and Dengue-1. Cultures and labeling are the same described in Fig. 3. A: gated CD14+ peripheral blood mononuclear leukocytes. Histograms represent gated CD14+ from (B) uninfected or (C) infected cells in which $x$-axis represent intensity of TRITC labeling specific for Dengue-1. M1 is a marker for the positive cell population.

\section{A}

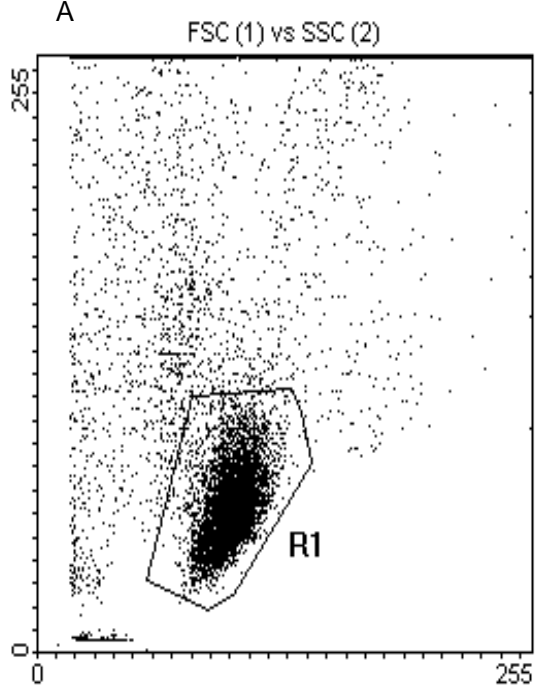

B

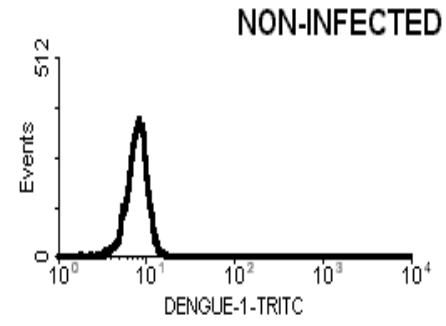

C

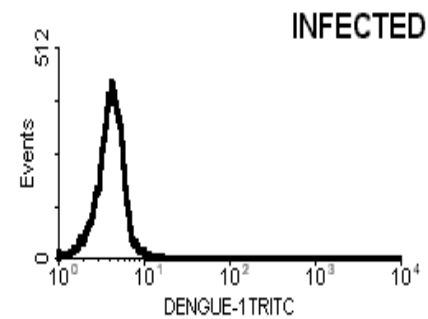

Fig. 5: histograms from gated lymphocytes obtained from fluorescent activated cell sorter analysis of Dengue-1 infected peripheral blood mononuclear leucocytes. Cultures and labeling are the same described in Fig. 3. A: gated lymphocytes according to their size and granularity ( $x$-axis, foward scattered cells (FSC) and $y$-axis, side scattered cells (SSC). Histograms represent gated lymphocytes from (B) uninfected or (C) five days infected cells in which $x$-axis represents intensity of labeling with TRITC specific for Dengue-1. M1 shows no positive cell population. 
TABLE

Frequency of positivity for Dengue-1 or Dengue-2 antigens in C6/36, Vero cells and adherent human peripheral blood mononuclear leukocytes (PBML). After five days of infection, specifically labeled cells were determined by fluorescent activated cell sorter analysis

\begin{tabular}{llccc}
\hline & Cell type & TCID $_{50}(\log )^{a}$ & $\begin{array}{c}\text { Virus } \\
(\% \text { IF cells })^{b}\end{array}$ & $\begin{array}{c}\text { Medium } \\
(\% \text { IF cells })\end{array}$ \\
\hline Dengue-1 & C6/36 & 6.20 & $63.21 \pm 3.1$ & $2.83 \pm 1.98$ \\
& Vero & 6.20 & $62.15 \pm 18.23$ & $4.75 \pm 1.79$ \\
& CD14+ D1+ ${ }^{c}$ & 7.20 & $27.23 \pm 0.07$ & $0.02 \pm 0.01$ \\
& CD14- D1+ & 7.20 & $1.05 \pm 0.58$ & $0.3 \pm 0.14$ \\
Dengue-2 & C6/36 & 6.95 & $90.93 \pm 1.03$ & $4.05 \pm 1.61$ \\
& Vero & 6.95 & $79.77 \pm 1.77$ & $2.4 \pm 0$ \\
& CD14+ D2+ & 7.95 & $23.56 \pm 0,13$ & $0,03 \pm 0,01$ \\
& CD14- D2+ & 7.95 & $0.9 \pm 0.3$ & $0.6 \pm 0$ \\
\hline
\end{tabular}

$a$ : virus load/well used for cell infection; $b: \%$ IF cells, represent percentage of cells with positive dengue specific immunofluorescence; $c$ : $2 \times 10^{5} \mathrm{C} 6 / 36$ or Vero cells were plated/well. Cells were sequentially labeled with antibody for Dengue-1 or Dengue-2 and anti-mouse Ig-FITC. Data from C6/36 and Vero cells are representative of three individual experiments each; $d: 2 \times 10^{6} \mathrm{PBML}$ were plated/well (about $10 \%$ are CD14+). Adherent PBMLs were double labeled with anti-CD14 prior to fixation and afterwards with antibody for Dengue-1 or Dengue- 2 and anti-mouse Ig-TRITC. Data from CD14+ cells are representative of two experiments using adherent cells, a third using total PBML cells infected with Dengue-1 and a fourth using total PBML cells infected with Dengue-2.

al. 1967) but counts of immunofluorescent focus has been recently used (Marianneau et al. 1998). Flow cytometry turned out to be a reliable methodology to quantify virus not only in cell line cultures such as C6/36 or Vero cells but also in PBML.

Flow cytometry has the advantage over RT-PCR techniques that it may count directly the rate of infected cells by antigen detection. Compared to traditional counts of fluorescent cells under the microscope, we have the intensity of fluorescence quantified per cell facilitating the distinction of specific and unspecific immunofluorescence and ruling out the possibility of subjective analysis. A greater number of cells are allowed to be counted, which is very important considering that in vitro viral infections are variable biological phenomena. This gives us the possibility of testing several replicates simultaneously and elevating the accuracy in statistical analysis. In case of cells that are not very susceptible, such as PBML, it is desired to count a large sample of negative cells in order to find a significant number of positive ones, turning manual counting very laborious. Kliks et al. (1989) quantified virus produced in infected monocyte cultures by plaque assay and correlated it with the counting of infected monocyte percentage detected by the immunofluorescent technique.

Recently one study reported rotaviruses detection in cell cultures by FACS using monkey cell line MA-104 (Barardi et al. 1998) and other authors detected dengue virus in endothelial cell lines (Avirutnan et al. 1998).
Barardi et al. (1998) used a different recover method as required for rotaviruses, trypsinizing the cells, which could eventually release surface molecules including dengue proteins. We detached cells from plates using cold medium and gentle scratch with plastic tips, which has been used for long to recover firmly adherent macrophages. We adapted well established techniques used to detect labile molecules, such as cytokines, and fixed cells with paraformaldehyde, instead of methanol which retains better cell characteristics and used saponin which provides milder permeabilization of membranes than Triton. With regard to dengue labeling, Avirutnan et al. (1998) used essentially the same reagents we did such as immune mouse ascitic fluid in an indirect immunofluorescent labeling detected by purified anti-mouse Igs conjugated to fluorochromes. It is important to note that blocking steps are highly desired especially for monocytes or other leukocytes but neither for mosquito C6/36 nor Vero cells.

Immunofluorescence of virus infected cells analyzed by FACS has shown to be sensitive and quantitative to detect extra and intracellular virus antigens and compare rates of infection in different cells. It may be used, for example, to evaluate of efficiency of seric antibodies to neutralize the infectivity of viruses or, additionally, to perform competitive studies determining cell receptors to virus entry in cells as well as relevant epitopes in cell penetration or in virus-cell interaction. 
This methodology may also provide us tools to compare different samples of wild viruses regarding to their capacity to infect macrophages/monocytes. Without an animal model to reproduce dengue disease, alternative assays have been searched to correlate virulence with clinic manifestations and disease severity. It will be also important to evaluate different sources of cells in primary cultures, which could potentially be target of dengue virus.

The virus load in CD14+ PBML is not high, which is in accord to earlier evidences that virus is not very frequently found in tissues from autopsies (Halstead 1988) and that the course of disease is short (a couple of weeks) and the disease finishes afterwards always without cronicity regardless of its benign or fatal outcome (Gubler 1998). It is believed that dengue virus is not per se cytopathic but it is triggering severe immunopathological reactions during infection (Halstead 1988). Therefore the study of interaction virus-host cells may lead to interesting and probably non-expected concepts of dengue pathology.

\section{ACKNOWLEDGEMENTS}

To Drs Marize Miagostovich and Pedro Borges for collaboration in virus titration.

\section{REFERENCES}

Anderson R, Wang S, Osiowy C, Isselutz AC 1997. Activation of endothelial cells via antibody-enhanced dengue virus infection of peripheral blood monocytes. J Virol 71: 4226-4232.

Avirutnan P, Malasit P, Seliger B, Bhakdi S, Husmann M 1998. Dengue infection of human endothelial cells leads to chemokine production, complement activation and apoptosis. J Immunol 161: 6338-6346.

Barardi CRM, Emslie KR, Vesey G, Willims KL 1998. Development of arapid and sensitive quantitative assay for rotavirus based om flow cytometry. $J$ Virol Meth 74: 31-38.

Bhamarapravati N Boonyapaknavik R 1966. Pathogenetic studies on thai haemorrhagic fever: Immunofluorescent localization on dengue virus in human tissue. Bull WHO 35: 50-51.

Boonpucknavig S, Boonpucknavig V, Bhamarapravati N, Nimmannitya S 1979. Immunofluorescence study of skin rash in patients with dengue hemorrhagic fever. Arch Pathol Lab Med 103: 464-466.

Brazilian National Health Foundation 1999. Ministry of Health, Repport from December 1999.

Deubel V 1997. The contribution of molecular techniques to the diagnosis of dengue infection. In DJ Gubler \& G Kuno (eds), Dengue and Dengue Hemorrhagic Fever, Cab International, Cambridge, p. 335-366.

Gubler DJ 1998. Dengue and dengue hemorrhagic fever. Clin Microb Rev 11: 480-496.

Halstead SB 1980. Immunological parameters of togaviruses disease syndromes. In RW Schlesinger, The Togaviruses. Biology, Structure, Replication, Academic Press, New York, p. 107-174.

Halstead SB 1988. Pathogenesis of dengue: challenges to molecular biology. Science 239: 476-481.

Igarashi A 1978. Isolation of a Singh's Aedes albopictus cell clone sensitive to dengue and chikungunya viruses. J Gen Virol 40: 531-544.

Kliks SC, Nisalak A, Brant WE, Wahl L, Burke DS 1989. Antibody-dependent enhancement of dengue virus growth in human monocytes as a risk factor for dengue hemorrhagic fever. Am J Trop Hyg 40: 444-451.

Kurane I, Janus J, Ennis FA 1992. Dengue virus infection of human skin fibroblasts in vitro production of IFN- $\beta$, IL-6 and GM-CSF. Arch Virol 124: 21-30.

Marianneau P, Steffan AM, Royer C, DrouetMT, Kirn A, Deubel V 1998. Differeing infection patterns of dengue and yellow fever viruses in human hepatoma cell line. J Infec Dis 178: 1270-1280.

Miagostovich MP, Nogueira RMR, Cavalcanti SMB, Marzochi KBF, Schatzmayr HG 1993. Dengue epidemic in the state of Rio de Janeiro, Brazil: virological and epidemiological aspects. Rev Inst Med Trop São Paulo 35: 149-154.

Morens DM, Marchette NJ, Chu MC, Halstead SH 1991. Growth of Dengue type 2 virus isolates in human peripheral blood leukocytes correlates with severe and mild dengue disease. Am J Trop Med Hyg 45: 644-651.

PAHO-Pan American Health Organization 1994. Dengue and dengue hemorrhagic fever in Americas: guidelines for prevention and control. PAHO/WHO (Scientific Publication N\#548), Washington, D.C.

Pang T 1987. Dengue haemorrhagic fever: virus or response? Bio Essays 6: 141-143.

Reed LJ, Muench H 1938. A simple method of estimating fifty per cent endpoints Am J Hyg 27: 493-497.

Russell PK, Nisalak A, Sukhavachana P, Vivona S 1967. A plaque reduction test for dengue virus neutralizing antibodies. J Immunol 99: 285-90.

Shoepp RJ, Beaty B 1984. Titration of dengue viruses by immunofluorescence in microtiter plates. $J$ Clin Microbiol 20: 1017-1019.

Vordam V, Kuno G 1997. Laboratory diagnosis of dengue virus infections. In DJ Gubler\& G Kuno (eds), Dengue and Dengue Hemorrhagic Fever, Cab International, Cambridge, p. 313-333. 